\title{
A sticky bacterium versus antiadhesive surfaces
}

Shogo Yoshimoto ${ }^{1}$, Ayane Kawashiri ${ }^{1}$, Taishi Matsushita ${ }^{2}$, Satoshi Ishii ${ }^{1}$, Stephan Göttig ${ }^{3}$, Volkhard A. J. Kempf ${ }^{3}$, Madoka Takai ${ }^{2}$, Katsutoshi Hori ${ }^{1 *}$

${ }^{1}$ Department of Biomolecular Engineering, Graduate School of Engineering, Nagoya University, Nagoya, Aichi 464-8603, Japan.

${ }^{2}$ Department of Bioengineering, Graduate School of Engineering, The University of Tokyo, Bunkyo-ku, Tokyo 133-8656, Japan.

${ }^{3}$ Institute for Medical Microbiology and Infection Control, University Hospital, Goethe University, Frankfurt 60596, Germany.

$10 \quad *$ Correspondence to: khori@chembio.nagoya-u.ac.jp

\begin{abstract}
While microorganisms have evolved to adhere and form biofilms on surfaces, various materials with antiadhesive surfaces have been developed. The Gram-negative bacterium Acinetobacter sp. Tol 5 exhibits high adhesiveness to various surfaces through AtaA, one of trimeric autotransporter adhesins (TAAs). We examined the adhesion of Tol 5 and other bacteria expressing different TAAs to antiadhesive surfaces. The results highlighted Tol 5's stickiness through AtaA, which enables cells to adhere even to antiadhesive materials including polytetrafluoroethylene with a low surface free energy, a hydrophilic polymer brush exerting steric hindrance, and mica with an ultrasmooth surface. Tol 5 cells also adhered to a zwitterionic 2-methacryloyloxyethyl-phosphorylcholine-polymer-coated surface but were exfoliated by a weak shear stress, suggesting that exchangeable bound water molecules contribute to AtaA's interaction with materials.
\end{abstract}

One Sentence Summary: Acinetobacter sp. Tol 5 can adhere to typical antiadhesive surfaces but its preference suggests an adhesion mechanism involving water molecules. 
Main Text: Pathogenic bacteria, like viruses, cause infectious diseases, and the threat is reminded by the COVID-19 pandemic. However, bacteria are not as much of a threat as viruses because antibiotics are effective against them. This is changing, however, with the emergence of antibiotic-resistant bacteria. The global expansion of multidrug-resistant bacteria has become a clinical problem (1), and the threat of bacterial infection might come back in the near future. The overuse of antibiotics amplifies the opportunity for resistant bacteria to emerge and spread (2). The increased antibiotic use during this COVID-19 pandemic could also increase the threat of resistant bacteria (3). As an alternative to antibiotics, antiadhesive (antibiofouling) surfaces have drawn intensive research interest because bacterial adhesion is the initial step of infection by pathogens and biofouling of equipment (4-7). As a result of extensive efforts, various antiadhesive surfaces have been developed and characterized, such as fluoropolymers, polymer brushes, highly hydrophilic zwitterionic polymers, and ultrasmooth or nano/micro-topographical patterned surfaces $(8-12)$.

Acinetobacter sp. Tol 5, which is a toluene-degrading bacterium that we previously isolated from a biofiltration system, exhibits autoagglutination and high adhesiveness to solid surfaces (13, 14). Tol 5 cells quickly adhere to various material surfaces from hydrophobic plastics to hydrophilic glasses and metals independently of biofilm formation (13). This characteristic nonspecific adhesiveness of Tol 5 cells is mediated by AtaA, a member of the trimeric autotransporter adhesin (TAA) family (15-17). TAAs are outer membrane proteins of Gramnegative bacteria and have been well-studied as virulence factors because each TAA shows an ability to bind to biotic molecules of mammalian host cells and occasionally to some kinds of abiotic surfaces $(18,19)$. Although they have a variety of lengths from several hundreds to several thousands of amino acids, they have a common structure that includes an N-terminal passenger domain (PSD), which is secreted onto the cell surface and is responsible for its function, and a C-terminal transmembrane domain, which anchors the PSD onto the outer membrane (19). AtaA is one of the largest TAAs consisting of 3,630 amino acids but shares common structural features with other TAAs, $(15,20)$. However, there have been no reports of TAA-mediated adhesion similar to Tol 5 cells through AtaA in terms of nonspecificity and high stickiness.

In a proverb known as the "shield-spear contradiction" derived from an ancient Chinese text Han Feizi, a merchant first boasts that, "this shield is strong enough to prevent anything," and then, 
"this spear is sharp enough to pierce anything." In response to the merchant's boasting, one person from the crowd asks the merchant, "What would happen if you attack your shield with your spear?" The merchant could not answer. Similarly, we also don't know what would happen if highly adhesive Tol 5 cells encounter an antiadhesive surface. In this study, we investigated the interaction of Tol 5 and some other TAA-expressing bacterial cells with various surfaces including antiadhesive surfaces that have different repelling mechanisms.

First, we compared the adhesiveness of Tol 5 and its $\triangle a t a A$ mutant (negative control) with that of Yersinia enterocolitica and Bartonella henselae by shaking each cell suspension in the presence of a polyurethane support for $30 \mathrm{~min}$. These Gram-negative bacteria have also been reported to adhere to abiotic surfaces through their TAAs (18), YadA and BadA, respectively. The production of these TAAs was confirmed by western blotting (see Supplementary Figure S1). The result showed the overwhelming stickiness of cells expressing AtaA compared with that of cells expressing the other TAAs (Fig. 1). Most of the Tol 5 cells adhered to the support and the cell suspension became abundantly clear. In contrast, the cell suspensions of $Y$. enterocolitica, B. henselae, and Tol $5 \Delta a t a A$ mutant remained cloudy, which indicated that many of the cells did not adhere to the polyurethane support.

Next, we quantified the adhesiveness of bacterial cells that express TAAs to various material surfaces. Cell suspensions were placed and incubated on polystyrene (PS), glass, stainless steel, and polytetrafluoroethylene (PTFE, known as Teflon) surfaces for $10 \mathrm{~min}$. Non-adhering cells were removed by washing with a fresh medium and the adhered cells on the material surface were quantified by crystal violet staining. As shown in Figure 2, in a short time (10 min), Tol 5 could adhere to not only PS, glass, and stainless steel but also to PTFE, which has antiadhesive properties derived from its low surface energy (8). In contrast, Tol $5 \Delta a t a A$ mutant and $Y$. enterocolitica hardly adhered to all the material surfaces. Although B. henselae showed measurable adhesiveness, the amount of adhered cells was much smaller than that of Tol 5. These results quantitatively demonstrated that Tol 5 cells exhibit remarkably higher adhesiveness to various material surfaces through AtaA than bacterial cells expressing other TAAs.

To investigate whether Tol 5 cells adhere to various other antiadhesive surfaces in addition to PTFE, we performed adhesion assays with mica, poly(oligo(ethylene glycol) methyl ether methacrylate) (poly(mOEGMA)) brush, and 2-methacryloyloxyethyl phosphorylcholine (MPC) 
polymer surfaces. Mica is a phyllosilicate mineral of aluminum and potassium, and its surface after cleaving is atomically flat (21). A poly(mOEGMA) brush is a neutral hydrophilic polymer brush and exerts steric repulsion (10). An MPC polymer is a zwitterionic hydrophilic polymer and possesses a high free water fraction (22). These surfaces have been reported to have antiadhesive properties against bacterial cells $(9,11,23)$. After incubation of bacterial cells on the antiadhesive surfaces for $10 \mathrm{~min}$, Tol 5 cells adhered to PTFE but not to the mica, poly(mOEGMA) brush, and MPC polymer surface (Fig. 3A). After incubation for $2 \mathrm{~h}$, Tol 5 cells adhered to not only PTFE but also to the mica and poly(mOEGMA) brush surface, but hardly adhered to the MPC polymer (Fig. 3A). In contrast, B. henselae adhered to PTFE and mica but not to the poly(mOEGMA) brush and the MPC polymer (Fig. 3B). These results emphasize that Tol 5 cells were the only cells that adhered to the poly(mOEGMA) brush and showed that even sticky Tol 5 cells hardly adhered to the MPC polymer under these experimental conditions.

To investigate how the MPC polymer repels Tol 5 cells, we observed the behavior of Tol 5 cells 15 on the polymer surface by using a flow cell system with a square glass tube (Fig. 4A) (24). The glass tube with or without the MPC polymer coating was filled with a Tol 5 cell suspension and incubated for $10 \mathrm{~min}$. Then, the cell suspension was replaced with fresh BS-N medium by slow flowing at $10 \mu \mathrm{L} / \mathrm{min}$ for rinsing, and the flow rate was increased stepwise, as shown in Figure $4 \mathrm{~B}$, while observing the inner surface of the bottom of the glass tube under a microscope. Unexpectedly, Tol 5 cells adhered to the MPC-polymer-coated glass as much as the bare (noncoated) glass under static conditions and remained adhered after rinsing at $10 \mu \mathrm{L} / \mathrm{min}$ (Fig 4C initial). When the flow rate was increased to $20 \mu \mathrm{L} / \mathrm{min}$, a small fraction of previously adhered cell clumps started to move and slip on the surface (see Supplementary Movie S1), but many cells still resisted detachment after $10 \mathrm{~min}$ of flowing (Fig. 4C, $20 \mu \mathrm{L} / \mathrm{min}$ ). At a high flow rate of $50 \mu \mathrm{L} / \mathrm{min}$ or more, the Tol 5 cells firmly adhered to the bare glass whereas the cells attached on the MPC polymer were exfoliated, rolled, and washed off from the surface by the shear stress ( $\geq 5.94 \mathrm{mN} / \mathrm{m}^{2}$ ) (Fig. 4C, $\geq 50 \mu \mathrm{L} / \mathrm{min}$ and see Supplementary Movie S1).

So far, various antiadhesive materials have been developed on the basis of repelling mechanisms. Fluoropolymers with a low surface free energy are widely used in cookware and medical equipment although their hydrophobicity is also said to cause protein adsorption that hiders cell attachment by contraries (25). Polymer brushes with a high grafting density have been especially 
studied as powerful antiadhesive surfaces for cell adhesion (26). However, the finding that Acinetobacter sp. Tol 5 is able to adhere to these antiadhesive materials makes us realize the marvel of microbial diversity and evolution. In addition, AtaA could mediate cell adhesion to poly(mOEGMA) brush but BadA could not. Note that BadA is similar to AtaA in size and abundance on the cell surface; it consists of 3,082 amino acids and its fibrous molecules peritrichately cover over bacterial cells (18). Therefore, their difference in adhesiveness demonstrates the functional diversity of the TAA family as a result of protein evolution.

Tol 5 cells even adhered to an MPC-polymer-coated surface but their interaction was so weak that the cells could be exfoliated by a weak shear stress. The exfoliated and rolling cell clumps seemed to involve and remove cell clumps that were still adhered owing to the autoagglutinating property of Tol 5 cells (24), self-cleaning the surface coated with the MPC polymer. In an adhesion assay using a microwell, the Tol 5 cells should have been detached by the washing step. MPC is a methacrylate monomer with a phosphorylcholine (PC) group, which is a hydrophilic polar head group of phospholipids comprising a eukaryotic cell membrane (27). MPC polymers are known to significantly suppress adhesion of proteins and cells because there are lots of free water molecules (22) but capture few bound water molecules on their PC group (27-29). The fact that Tol 5 cells can adhere to the poly(mOEGMA) brush and the mica, but can only interact very weakly with a surface coated with MPC polymers, despite similar levels of hydrophilicity, as shown by the static contact angles of air in water (Table S2), suggests that exchangeable bound water molecules contribute to the interaction between AtaA and material surfaces (28).

\section{References and Notes}

1. S. Baker, N. Thomson, F. X. Weill, K. E. Holt, Genomic insights into the emergence and spread of antimicrobial-resistant bacterial pathogens. Science 360, 733-738 (2018).

2. "WHO report on surveillance of antibiotic consumption: 2016-2018 early implementation," World Health Organization (2018).

3. J. Hsu, How covid-19 is accelerating the threat of antimicrobial resistance. BMJ 369, m1983 (2020).

4. I. Banerjee, R. C. Pangule, R. S. Kane, Antifouling coatings: Recent developments in the design of surfaces that prevent fouling by proteins, bacteria, and marine organisms. $A d v$ Mater 23, 690-718 (2011). 
5. C. Berne, C. K. Ellison, A. Ducret, Y. V. Brun, Bacterial adhesion at the single-cell level. Nat Rev Microbiol 16, 616-627 (2018).

6. L. Hall-Stoodley, J. W. Costerton, P. Stoodley, Bacterial biofilms: From the natural environment to infectious diseases. Nat Rev Microbiol 2, 95-108 (2004).

7. K. Hori, S. Matsumoto, Bacterial adhesion: From mechanism to control. Biochem Eng J 48, 424-434 (2010).

8. C. I. Pereni, Q. Zhao, Y. Liu, E. Abel, Surface free energy effect on bacterial retention. Colloid Surface B 48, 143-147 (2006).

9. G. Cheng, Z. Zhang, S. F. Chen, J. D. Bryers, S. Y. Jiang, Inhibition of bacterial adhesion and biofilm formation on zwitterionic surfaces. Biomaterials 28, 4192-4199 (2007).

10. M. C. Sin, S. H. Chen, Y. Chang, Hemocompatibility of zwitterionic interfaces and membranes. Polym J 46, 436-443 (2014).

11. G. H. Zeng, T. Muller, R. L. Meyer, Single-cell force spectroscopy of bacteria enabled by naturally derived proteins. Langmuir 30, 4019-4025 (2014).

12. J. Hasan, K. Chatterjee, Recent advances in engineering topography mediated antibacterial surfaces. Nanoscale 7, 15568-15575 (2015).

13. M. Ishikawa, K. Shigemori, A. Suzuki, K. Hori, Evaluation of adhesiveness of Acinetobacter sp. Tol 5 to abiotic surfaces. J Biosci Bioeng 113, 719-725 (2012).

14. K. Hori, S. Yamashita, S. Ishii, M. Kitagawa, Y. Tanji, H. Unno, Isolation, characterization and application to off-gas treatment of toluene-degrading bacteria. J Chem Eng Jpn 34, 1120-1126 (2001).

15. M. Ishikawa, H. Nakatani, K. Hori, AtaA, a new member of the trimeric autotransporter adhesins from Acinetobacter sp. Tol 5 mediating high adhesiveness to various abiotic surfaces. PLoS One 7, e48830 (2012).

16. K. Noba, M. Ishikawa, A. Uyeda, T. Watanabe, T. Hohsaka, S. Yoshimoto, T. Matsuura, K. Hori, Bottom-up creation of an artificial cell covered with the adhesive bacterionanofiber protein AtaA. J Am Chem Soc 141, 19058-19066 (2019).

17. S. Yoshimoto, H. Nakatani, K. Iwasaki, K. Hori, An Acinetobacter trimeric autotransporter adhesin reaped from cells exhibits its nonspecific stickiness via a highly stable 3D structure. Sci Rep 6, 28020 (2016). 
18. N. F. Muller, P. O. Kaiser, D. Linke, H. Schwarz, T. Riess, A. Schafer, J. A. Eble, V. A. J. Kempf, Trimeric autotransporter adhesin-dependent adherence of Bartonella henselae, Bartonella quintana, and Yersinia enterocolitica to matrix components and endothelial cells under static and dynamic flow conditions. Infect Immun 79, 2544-2553 (2011).

19. D. Linke, T. Riess, I. B. Autenrieth, A. Lupas, V. A. J. Kempf, Trimeric autotransporter adhesins: variable structure, common function. Trends Microbiol 14, 264-270 (2006).

20. K. Koiwai, M. D. Hartmann, D. Linke, A. N. Lupas, K. Hori, Structural basis for toughness and flexibility in the C-terminal passenger domain of an Acinetobacter trimeric autotransporter adhesin. J Biol Chem 291, 3705-3724 (2016).

21. W. de Poel, S. Pintea, J. Drnec, F. Carla, R. Felici, P. Mulder, J. A. A. W. Elemans, W. J. P. van Enckevort, A. E. Rowan, E. Vlieg, Muscovite mica: Flatter than a pancake. Surf Sci 619, 19-24 (2014).

22. K. Ishihara, H. Nomura, T. Mihara, K. Kurita, Y. Iwasaki, N. Nakabayashi, Why do phospholipid polymers reduce protein adsorption? J Biomed Mater Res 39, 323-330 (1998).

23. T. Kameda, K. Ohkuma, S. Oka, Polytetrafluoroethylene (PTFE): A resin material for possible use in dental prostheses and devices. Dent Mater J 38, 136-142 (2019).

24. Y. Furuichi, K. Iwasaki, K. Hori, Cell behavior of the highly sticky bacterium Acinetobacter sp. Tol 5 during adhesion in laminar flows. Sci Rep 8, 8285 (2018).

25. Z. K. Zander, M. L. Becker, Antimicrobial and antifouling strategies for polymeric medical devices. ACS Macro Lett 7, 16-25 (2018).

26. M. Krishnamoorthy, S. Hakobyan, M. Ramstedt, J. E. Gautrot, Surface-initiated polymer brushes in the biomedical field: Applications in membrane science, biosensing, cell culture, regenerative medicine and antibacterial coatings. Chem Rev 114, 10976-11026 (2014).

27. Y. Iwasaki, K. Ishihara, Cell membrane-inspired phospholipid polymers for developing medical devices with excellent biointerfaces. Sci Technol Adv Mat 13, 064101 (2012).

28. D. Nagasawa, T. Azuma, H. Noguchi, K. Uosaki, M. Takai, Role of interfacial water in protein adsorption onto polymer brushes as studied by SFG spectroscopy and QCM. J Phys Chem C 119, 17193-17201 (2015).

29. T. Azuma, R. Ohmori, Y. Teramura, T. Ishizaki, M. Takai, Nano-structural comparison of 2-methacryloyloxyethyl phosphorylcholine- and ethylene glycol-based surface 
modification for preventing protein and cell adhesion. Colloid Surface B 159, 655-661 (2017).

30. Y. Y. Lu, B. Franz, M. C. Truttmann, T. Riess, J. Gay-Fraret, M. Faustmann, V. A. J. Kempf, C. Dehio, Bartonella henselae trimeric autotransporter adhesin BadA expression interferes with effector translocation by the VirB/D4 type IV secretion system. Cell Microbiol 15, 759-778 (2013).

31. T. Riess, S. G. E. Andersson, A. Lupas, M. Schaller, A. Schafer, P. Kyme, J. Martin, J. H. Walzlein, U. Ehehalt, H. Lindroos, M. Schirle, A. Nordheim, I. B. Autenrieth, V. A. J. Kempf, Bartonella adhesin A mediates a proangiogenic host cell response. J Exp Med 200, 1267-1278 (2004).

32. K. Hori, Y. Ohara, M. Ishikawa, H. Nakatani, Effectiveness of direct immobilization of bacterial cells onto material surfaces using the bacterionanofiber protein AtaA. Appl Microbiol Biotechnol 99, 5025-5032 (2015).

33. H. Watanabe, Y. Tanji, H. Unno, K. Hori, Rapid conversion of toluene by an Acinetobacter sp. Tol 5 mutant showing monolayer adsorption to water-oil interface. J Biosci Bioeng 106, 226-230 (2008).

34. M. Ishikawa, K. Hori, A new simple method for introducing an unmarked mutation into a large gene of non-competent Gram-negative bacteria by FLP/FRT recombination. BMC Microbiol 13, 86 (2013).

35. M. Drancourt, R. Birtles, G. Chaumentin, F. Vandenesch, J. Etienne, D. Raoult, New serotype of Bartonella henselae in endocarditis and cat-scratch disease. Lancet 347, 441443 (1996).

36. J. Heesemann, R. Laufs, Construction of a mobilizable Yersinia enterocolitica virulence plasmid. J Bacteriol 155, 761-767 (1983).

Acknowledgments: We thank Kai lio and Eriko Matsui for the adhesion assay. Funding: This work was supported by the Japan Society for the Promotion of Science (JSPS) KAKENHI (Grant Numbers JP17H01345, JP18K14062) and by the Deutsche Forschungsgemeinschaft (DFG research group 2251). Author contributions: K.H. designed the study, and S.Y., A.K., and K.H. wrote the paper. S.Y. and A.K. conducted the adhesion assay. A.K., T.M., and M.D. prepared the poly(mOEGMA) brush surface and measured the static contact angles of air bubbles in water. 
S.I. and A.K. conducted cultivation and protein analysis of bacterial cells with help from S.G. and V.A.J.K. All authors reviewed the manuscript. Competing interests: Authors declare no competing interests. Data and materials availability: All data are available in the main text or the supplementary materials.

\section{Supplementary Materials:}

Materials and Methods

Figures S1-S2

Tables S1-S2

Movie S1 
Tol 5

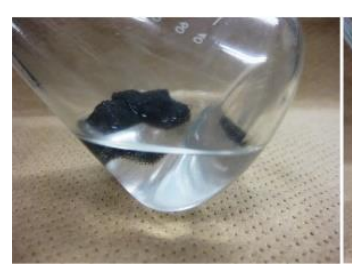

Tol $5 \Delta a t a A$

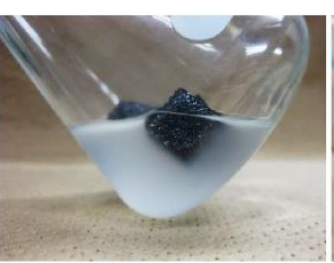

B. henselae

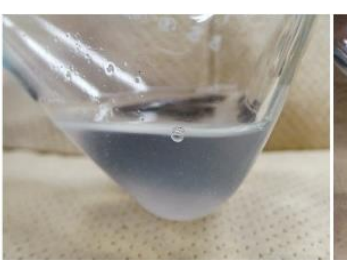

Y. enterocolitica

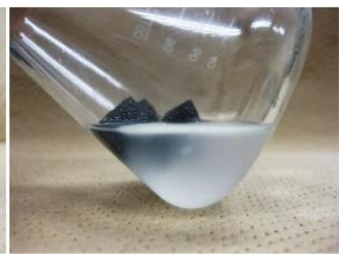

Fig. 1. Adhesion of bacterial cells to a polyurethane surface. Each panel shows the bacterial cell suspension after shaking for 30 min with a polyurethane foam support. 

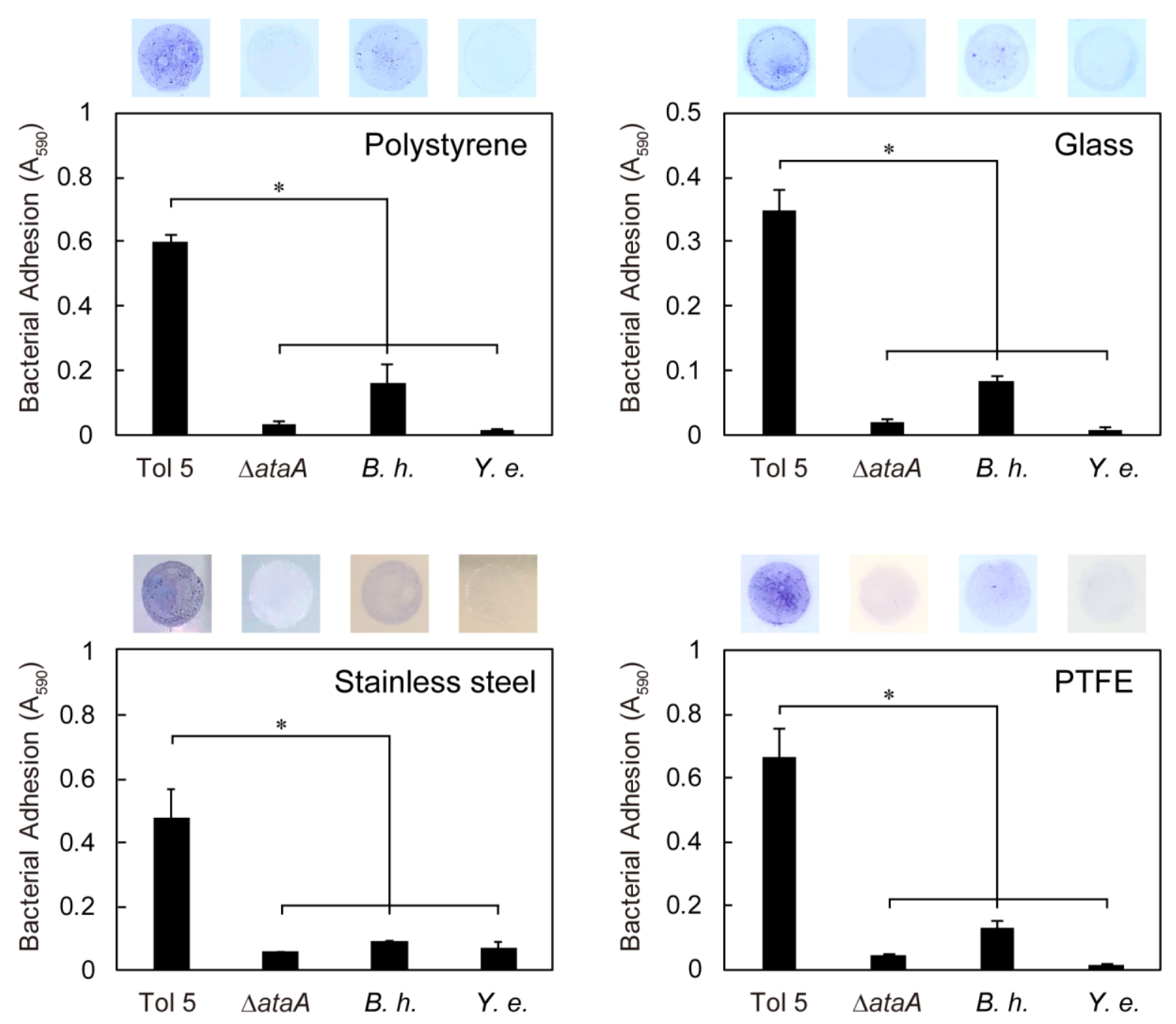

Fig. 2. Adhesion of bacterial cells to various materials. Adhesion of Acinetobacter sp. Tol 5, Tol $5 \triangle a t a A, B$. henselae (B. h.), and Y. enterocolitica (Y. e.) to polystyrene, glass, stainless steel, and PTFE was assessed by microwell adhesion assays for $10 \mathrm{~min}$. Data are expressed as the mean \pm SEM ( $\mathrm{n}=3)$. Significant differences from the result of Tol 5, analyzed using Student's t-test, are indicated by an asterisk $(\mathrm{p}<0.05)$. Upper photographs show the adhered cells on the material surfaces. 

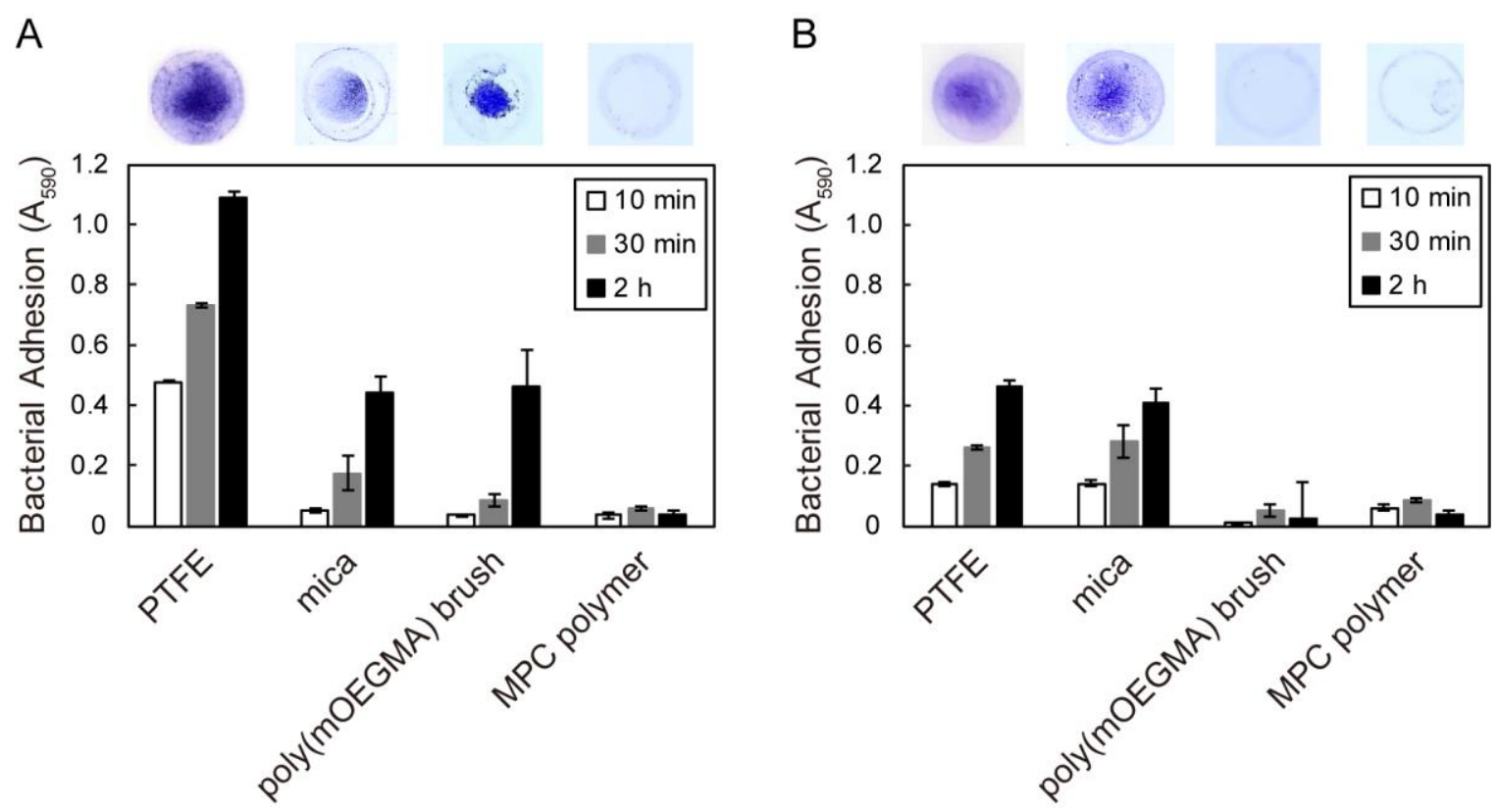

Fig. 3. Adhesion of bacterial cells to antiadhesive surfaces. Adhesion of Tol 5 (A) and $B$. henselae (B) to PTFE, mica, poly(mOEGMA) brush on glass, and MPC-polymer-coated glass, was assessed by microwell adhesion assays. Data are expressed as the mean \pm SEM $(n=3)$. Upper photographs show the adhered cells on the material surfaces after incubation for $2 \mathrm{~h}$. 
A

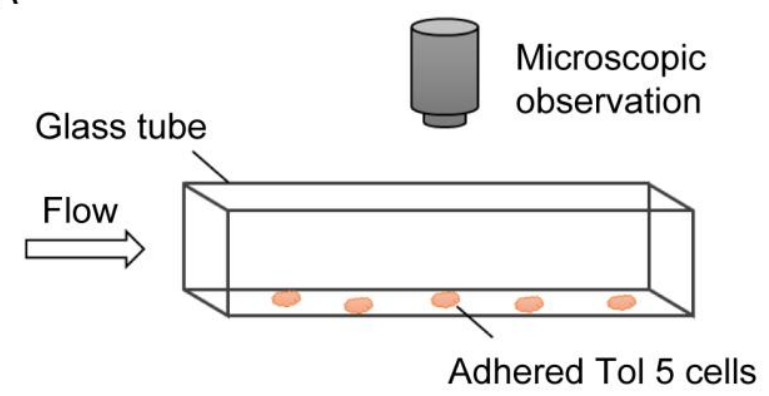

C

\begin{tabular}{|c|c|c|c|c|c|}
\hline $\begin{array}{c}\text { Flow rate } \\
(\mu \mathrm{l} / \mathrm{min})\end{array}$ & 10 & 20 & 50 & 100 & 200 \\
\hline $\begin{array}{l}\text { Shear stress } \\
\qquad\left(\mathrm{mN} / \mathrm{m}^{2}\right)\end{array}$ & 1.19 & 2.38 & 5.94 & 11.9 & 23.8 \\
\hline
\end{tabular}

MPC polymer

Bare glass
$\mathrm{B}$

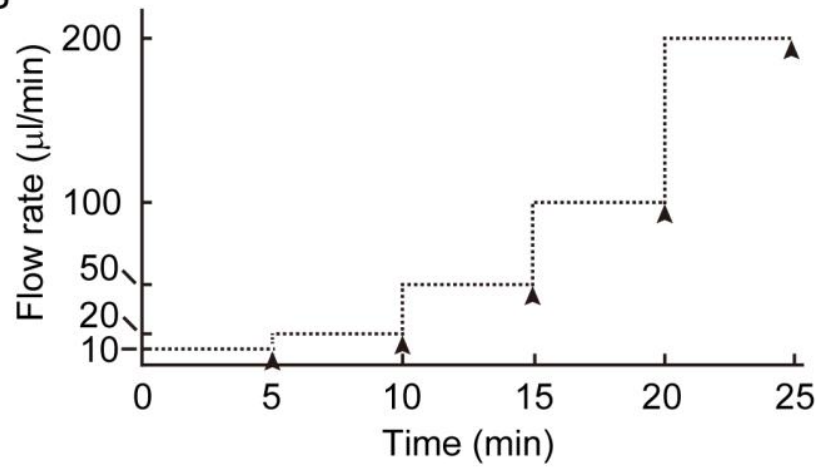

me $(\min )$

25

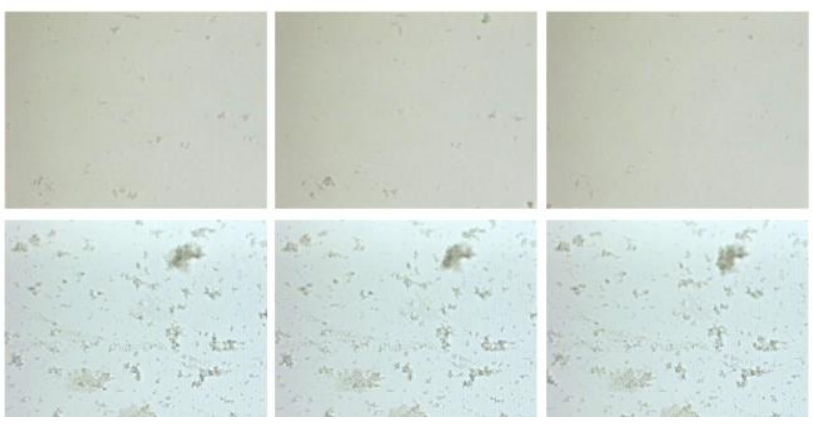

Fig. 4. Observation of the behavior of Tol 5 cells that were adhered to the MPC polymer surface beforehand. (A) Schematic representation of the flow cell system used in this study. (B) Transition of the flow rate. The flow rate was increased stepwise every 5 min. The black arrowheads indicate the time at which snapshots of the inner surface at the bottom of the glass tubes were captured. (C) The snapshots captured as described in (B). 